\title{
FATIGUE LIFE ASSESSMENT OF REACTOR COOLANT SYSTEM COMPONENTS BY USING TRANSFER FUNCTIONS OF INTEGRATED FE MODEL
}

\author{
SHIN-BEOM CHOI ${ }^{1}$, YOON-SUK CHANG ${ }^{2}$, JAE-BOONG CHOI ${ }^{1}$, YOUNG-JIN KIM ${ }^{1, *}$, \\ MYUNG-JO JHUNG ${ }^{3}$ and YOUNG-HWAN $\mathrm{CHOI}^{3}$ \\ ${ }^{1}$ School of Mechanical Engineering, Sungkyunkwan University \\ 300 Chunchun-dong, Jangan-gu, Suwon, Kyonggi-do 440-746, Republic of Korea \\ ${ }^{2}$ Department of Nuclear Engineering, Kyung Hee University \\ 1 Seocheon-dong, Giheung-gu, Yongin, Kyonggi-do 446-701, Republic of Korea \\ ${ }^{3}$ Korea Institute of Nuclear Safety \\ PO BOX 114, Yuseong-gu, Daejeon 305-600, Republic of Korea \\ *Corresponding author. E-mail : yjkim50@skku.edu
}

Received October 14, 2009

Accepted for Publication June 10, 2010

Recently, efficient operation and practical management of power plants have become important issues in the nuclear industry. In particular, typical aging parameters such as stress and cumulative usage factor should be determined accurately for continued operation of a nuclear power plant beyond design life. However, most of the major components have been designed via conservative codes based on a 2-D concept, which do not take into account exact boundary conditions and asymmetric geometries. The present paper aims to suggest an effective fatigue evaluation methodology that uses a prototype of the integrated model and its transfer functions. The validity of the integrated 3-D Finite Element (FE) model was proven by comparing the analysis results of individual FE models. Also, mechanical and thermal transfer functions, known as Green's functions, were developed for the integrated model with the standard step input. Finally, the stresses estimated from the transfer functions were compared with those obtained from detailed 3-D FE analyses results at critical locations of the major components. The usefulness of the proposed fatigue evaluation methodology can be maximized by combining it with an on-line monitoring system, and this combination, will enhance the continued operations of old nuclear power plants.

KEYWORDS : Continued Operation, Finite Element Method, Integrated Model, On-line Monitoring System, Transfer Functions

\section{INTRODUCTION}

With the Korean government declaring a green growth policy against global warming and the worldwide energy crisis, Nuclear Power Plants (NPPs) have returned back to the spotlight as a practical way for this policy to work. With this atmosphere, based on extensive technical and economical assessments, the nuclear industry has promoted the continued operation and practical management of existing plants as well as the construction of new plants. The foremost Korean continued operation of NPP started in 2008, which preludes of the Renaissance of nuclear power. However, extensive research must precede a safe continued operation because major components will have to sustain their functions beyond their design lives. In order to prevent unanticipated failures of major systems and/or components, for instance, and to assure the fatigue integrity of the Reactor Coolant System (RCS), state-of-the-art issues such as thermal stratification, environmental effects and so on have to be resolved.

The Fatigue Monitoring Program (FMP) [1, 2] is one of the efficient tools used to predict damages of major components caused by diverse operating modes and environments. In USA, several commercial FMPs have been installed in NPPs. Especially, EPRI developed FatiguePro [3] which is able to assess fatigue life with real-time operating data. Also, Operating Transients Monitoring System (OTMS) and Fatiguemeter [4] were developed in France and FAtigue MOnitoring System (FAMOS) was developed in Germany to determine the aging status of operating NPPs. Although, Yoo et al. [5] developed K-FAMS in Korea, the well-known FatiguePro has been customized and installed in operating NPPs since 2005.

The aforementioned FMPs use Green's functions and manipulate real-time monitored data to calculate stresses. 
However, in general, Green's functions are derived from 2-D Finite Element (FE) analyses which cannot reflect exact boundary conditions and complex geometries. For this reasons, 2-D FE analyses may lead to conservative results and an unrealistic estimation of structural behavior. In cases dealing with complex geometries and operating data, 3-D modeling followed by FE analyses should be introduced to describe realistic structural behavior and predict a more accurate fatigue life. In the authors' previous studies [6-9], transfer functions of major components that comprise the RCS, such as the Reactor Pressure Vessel (RPV), Steam Generator (SG) and Pressurizer (PZR), were developed by considering performance and efficiency of contemporary computer technology. The objective of this paper is to suggest an effective fatigue evaluation methodology by using a prototype of integrated 3-D FE model and its transfer functions. To achieve this goal, the validity of the integrated model is determined by comparison of its results with those of $\mathrm{FE}$ analyses of individual models. Finally, thermal and mechanical stress transfer functions, also known as Green's functions, are derived from the integrated model with unit step inputs of temperature and pressure, respectively.

\section{FINITE ELEMENT ANALYSES}

\subsection{Individual Models and Integrated Model}

A prototype of an integrated FE model was developed by using a general-purpose mesh generating program, Hypermesh, which was used for stress analyses to derive transfer functions. For the modeling, a representative Korean NPP made up of one RPV, two SGs, four Reactor Coolant Pumps (RCPs), one PZR and main piping such as the hot leg, cold leg, cross-over leg and surge line, was chosen as depicted in Figure 1(a). An eight-node hexahedron element and four-node tetrahedron element were employed to construct the integrated FE model (element type Solid45 in ANSYS element library [10]). While the Solid45 elements were generated in the form of hexahedron and tetrahedron according to complex geometry, the same degree of freedom such as UX, UY and UZ was assigned to nodes that consisted of the integrated FE model. The integrated FE model consisted of 465,263 elements and 320,168 nodes, in which 9 critical locations were set: Point A (beltline region), Point B (bottom head to shell region) and Point $C$ (bottom head to shell transition region) were located in the RPV. For the PZR, Point D (the end point of surge nozzle), Point E (the bottom head to shell transition region) and Point F (shell region) were selected as the critical locations. Point $G$ (near the joint locations connected with the nozzle of hot leg), Point $\mathrm{H}$ (the safety nozzle) and Point I (complex geometry) were located in the SG.

On the other hand, to compare the stress distributions obtained from the integrated model, individual FE models of the RPV, SG and PZR were generated. The numbers of elements of the models ranged from 24,495 to 109,119 and the number of nodes ranged from 29,291 to 97,226 , respectively. Since these individual FE models were comparable to parts of the integrated FE model, mesh information and critical locations of each component were assumed to be the same.

\subsection{FE Model Verification}

In order to show the validity of the prototype of the integrated FE model, preliminary stress analyses were carried out by using the general-purpose finite element program, ANSYS. Then, the well-known Lame's equation was employed, which is applicable to a cylinder subjected to internal pressure only, for simple calculation of the corresponding reference hoop stress. The resulting hoop stress distributions in the inner surfaces, which is sufficiently far from the geometric discontinuities of RPV, PZR and $\mathrm{SG}$ of the integrated FE model, were in good agreement with the theoretical stress distributions. Since the maximum differences of the comparison results were less than $3 \%$, the integrated FE model was adopted to carry out detailed numerical analyses and to develop transfer functions. While diverse design transients have to be taken into account for calculation of the cumulative usage factors, in this paper, a typical transient of 'large turbine step load decrease' was selected to perform the stress analyses for brevity. Figure 2 depicts the pressure and temperature variation during the large turbine step load decrease [11].

\subsection{Component Materials}

The major components of a representative Korean NPP considered in this paper were made of several materials. Even though the most typical material, SA508 Gr.3 Cl.1 carbon steel, was used for the RPV, SG, RCP and PZR shell, other materials such as SA516 Gr.70 and SA312

Table 1. Materials of Major Components

\begin{tabular}{c|c}
\hline Components & Materials \\
\hline Reactor Pressure Vessel & SA508 Gr.3 Cl.1 \\
\hline Pressurizer & SA508 Gr.3 Cl.1(Shell) \\
SA541 Gr.3 Cl.1(Surge Nozzle) \\
\hline Steam Generator & SA508 Gr.3 Cl.1 \\
\hline Reactor Coolant Pump & SA508 Gr.3 Cl.1 \\
\hline Hot Leg & SA508 Gr.1A(Straight Pipe) \\
Cold Leg & SA516 Gr.70(Elbow) \\
\hline Cross Over Leg & SA312 TP347(Straight Pipe) \\
Surge Line & SA403 WP347(Elbow) \\
\hline
\end{tabular}


TP347 steels were also used for the surge nozzle as well as the main pipes comprised of straight pipes and elbows. The materials used for each component are summarized in Table 1.

\subsection{Loading and Boundary Conditions}

As loading condition, the time histories of pressure and temperature were applied as a distributed load to the inner surfaces of each component. In relation to the heat transfer analyses, a heat convection coefficient of $1.358 \mathrm{~W} / \mathrm{m}^{2} \cdot{ }^{\circ} \mathrm{C}$ was used. Also, the cladding effect was not considered to reduce the burden of numerical expenses, which resulted in relatively conservative temperature distributions.

For boundary conditions, the end parts of SG and RCP were fixed at all directions along with the Y-directional constraint for the shear lug of SG. All degree of freedom

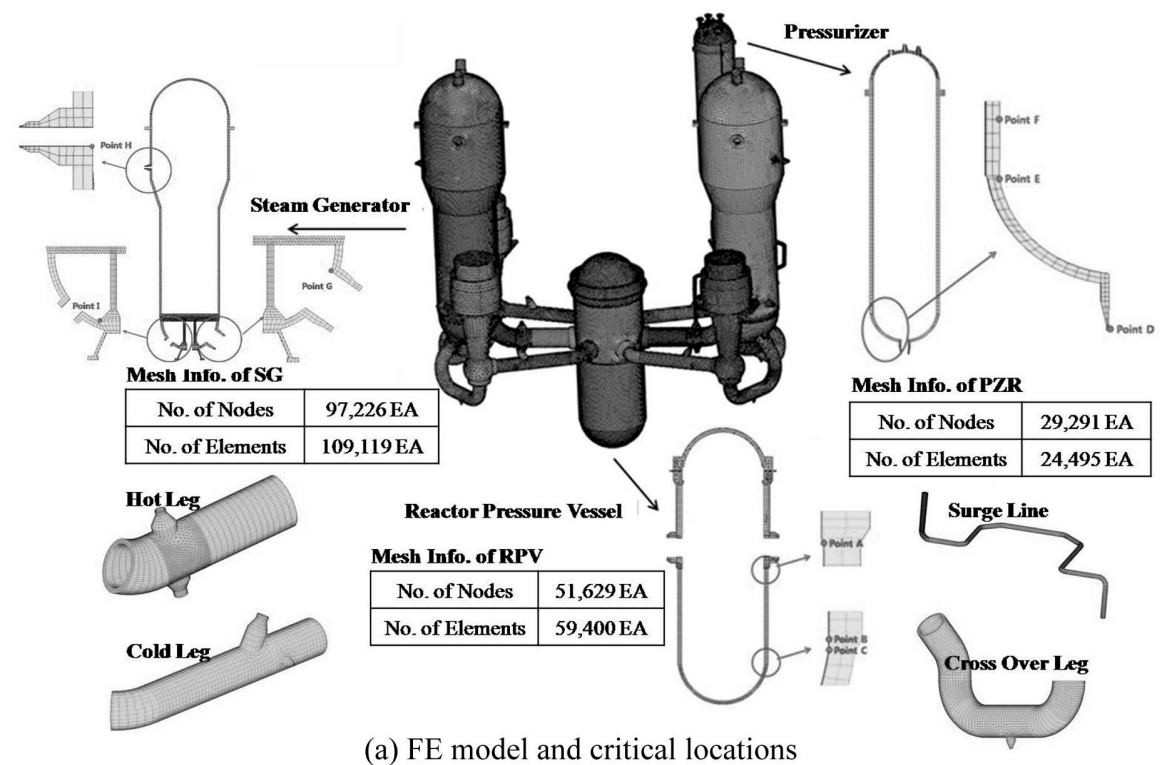

(a) FE model and critical locations

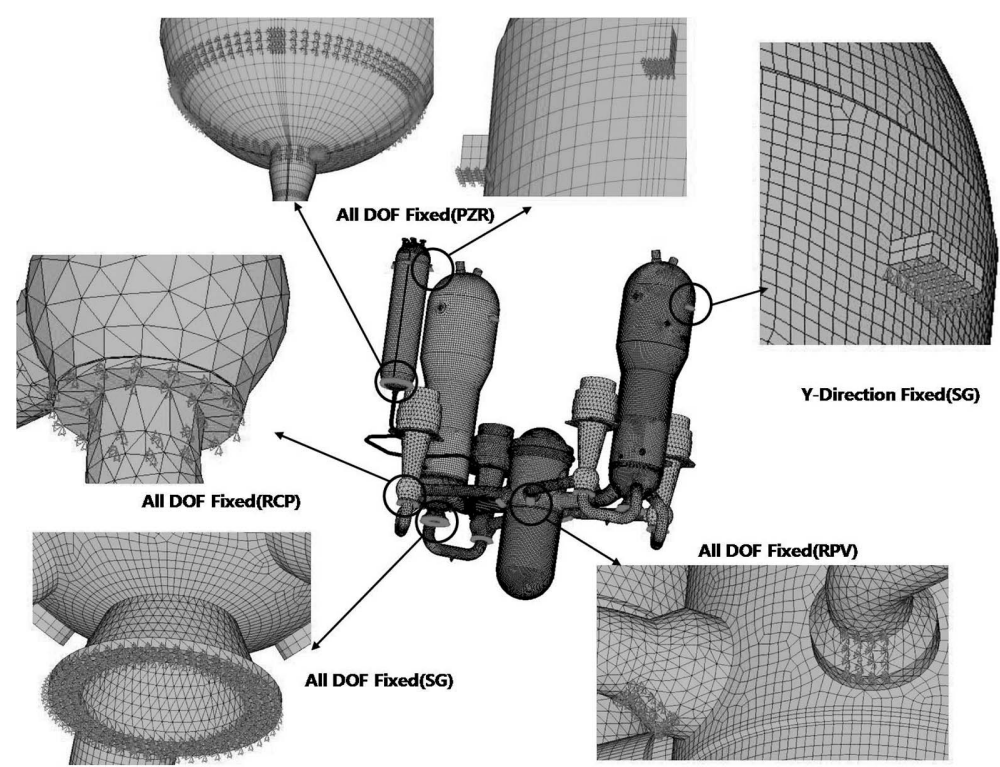

(b) Boundary conditions of integrated model

Fig. 1. FE Model, Critical Locations and Boundary Conditions of Integrated Model 


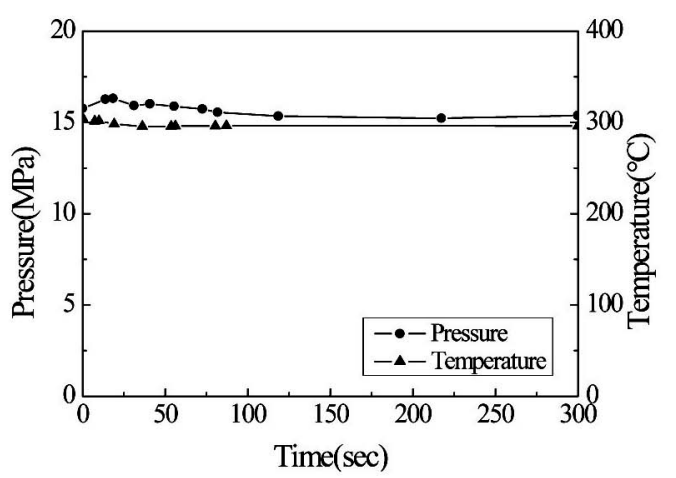

RPV

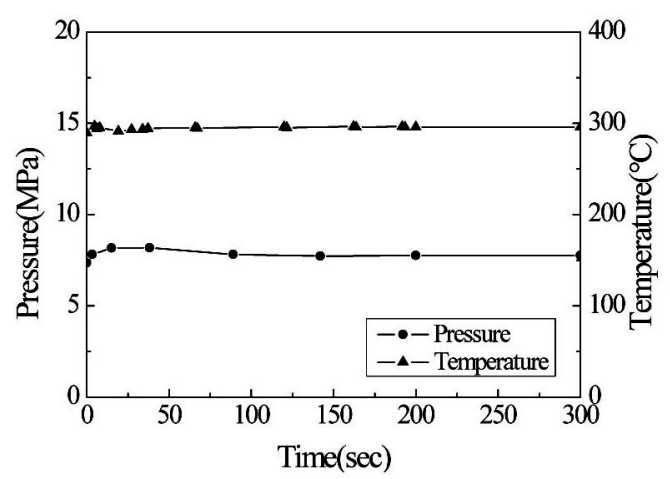

SG

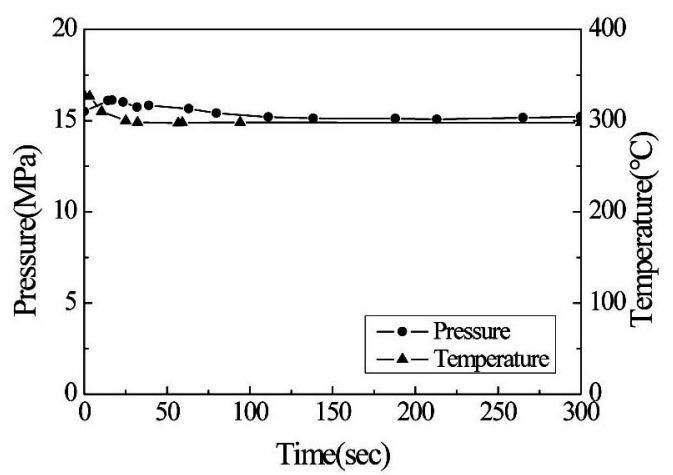

Hot leg

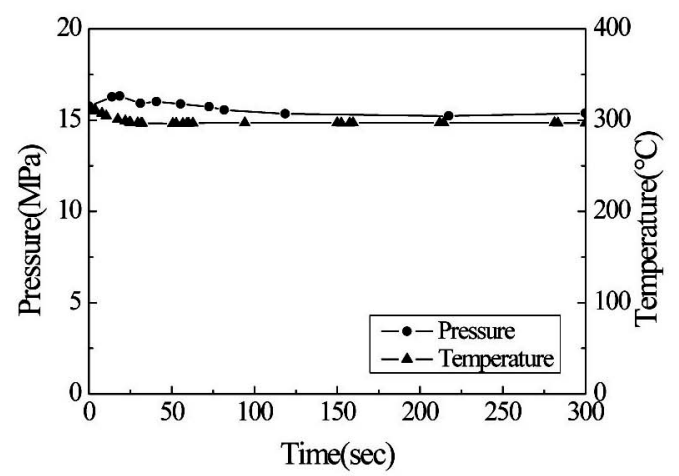

Cross over leg

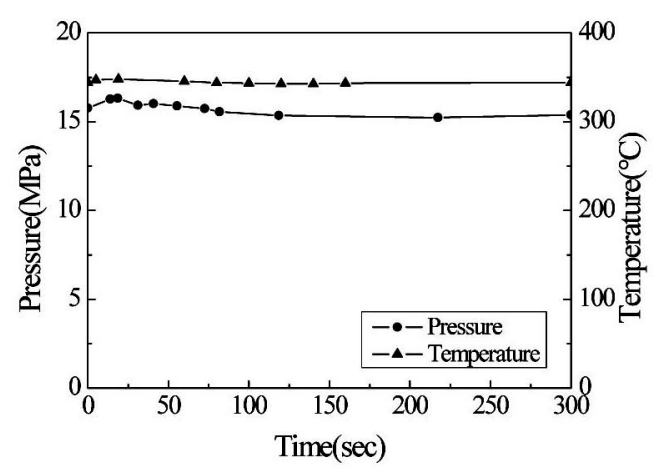

PZR

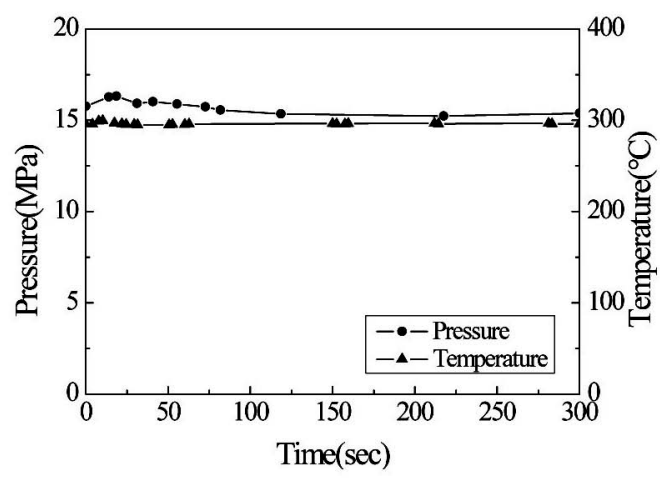

RCP

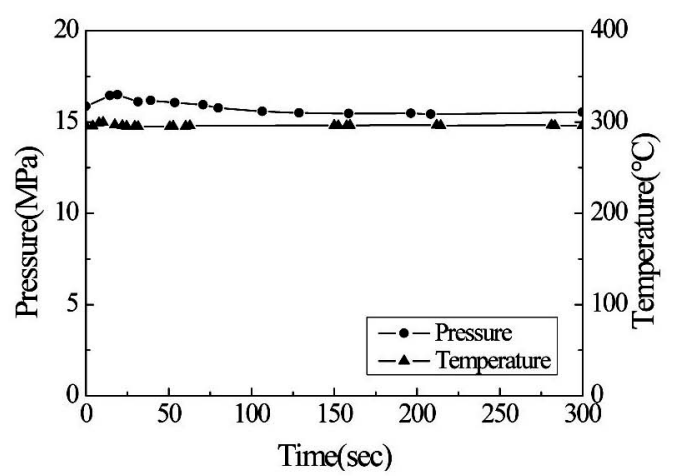

Cold leg

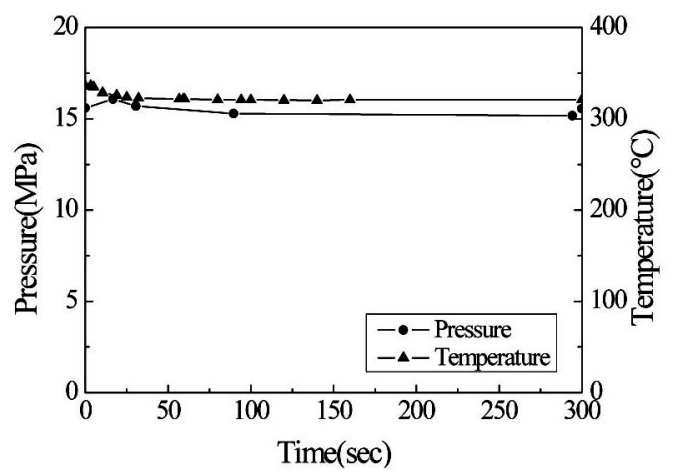

Surge line

Fig. 2. The Time Histories of Pressure and Temperature During Large Turbine Step Load Decrease 
at the shear lug and skirt region of the PZR were fixed instead of detailed modeling since this research centered on the main bodies of RCS major components. Regarding to the RPV, four nozzles were connected with safety plates. In reality, the safety plates were not modeled because they were not considered to be the part of critical locations. Instead of the detailed modeling of them, all the degree of freedom from RPV's four nozzles was constrained. Figure 1(b) shows boundary conditions of the integrated model.

\subsection{FE Analysis Results and Discussions}

The von Mises effective stresses obtained from each critical location of the integrated model were compared with those obtained from the individual models, as depicted in Figure 3. The stresses were in good agreement at most of the critical locations except for Points A, D, G and I. The disagreements at Points A, D, G and I can be best explained by considering that the Points $\mathrm{A}$ and I were near the joint locations that were connected with the hot leg and nozzles of the RPV and SG. Point G was near the joint location connected between the nozzle of hot leg and that of SG. Point D was also the location of connection between the surge line and surge nozzle of the PZR, so it has material discontinuity and complex geometry. Another reason for disagreement is the discordance between the

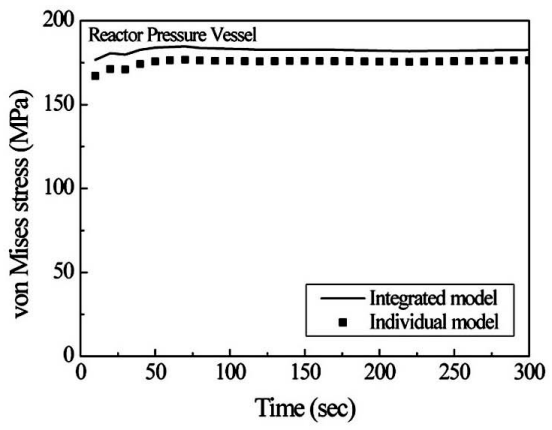

Point A

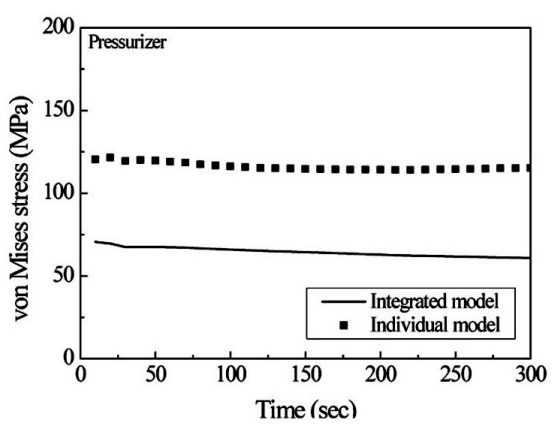

Point D

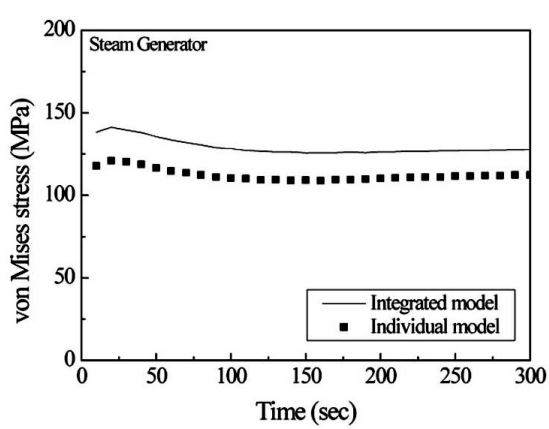

Point $\mathrm{G}$

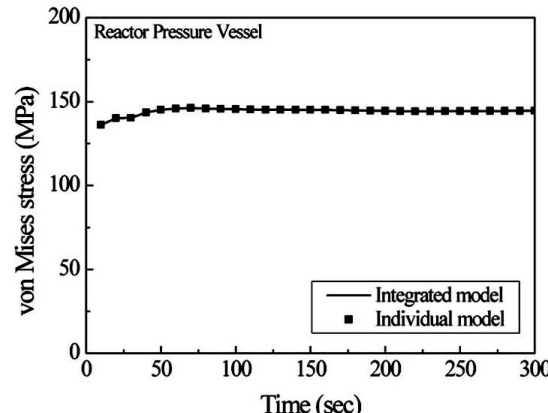

Point B

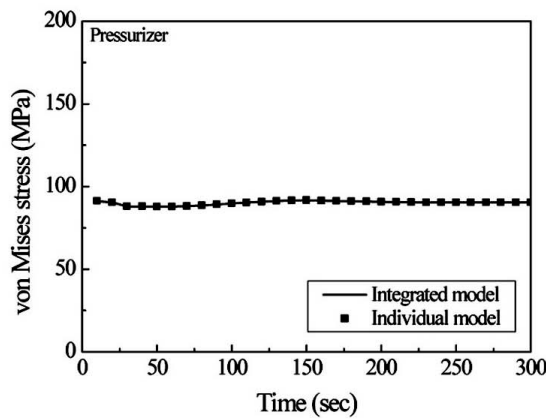

Point E

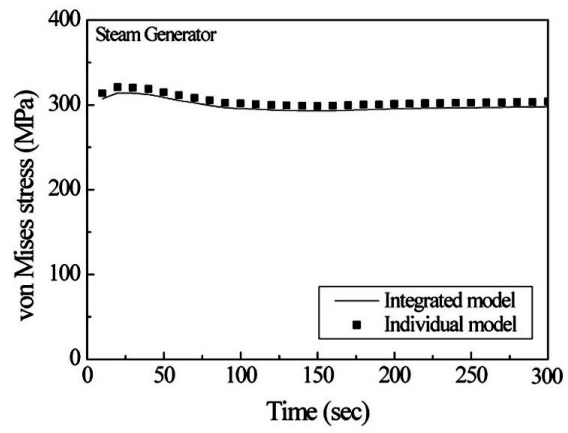

Point $\mathrm{H}$

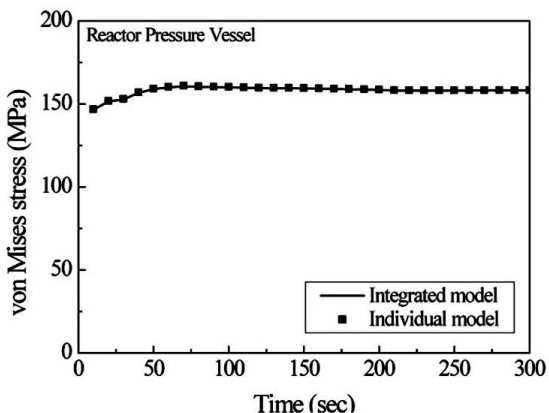

Point C

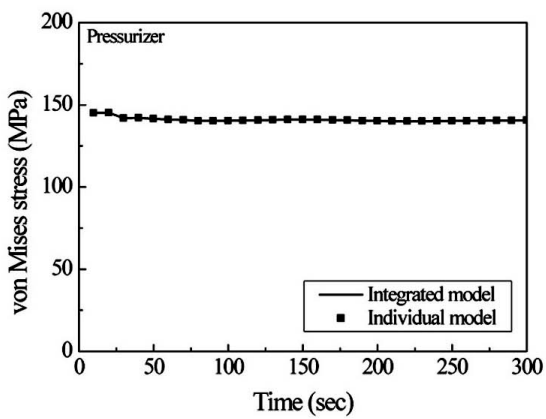

Point F

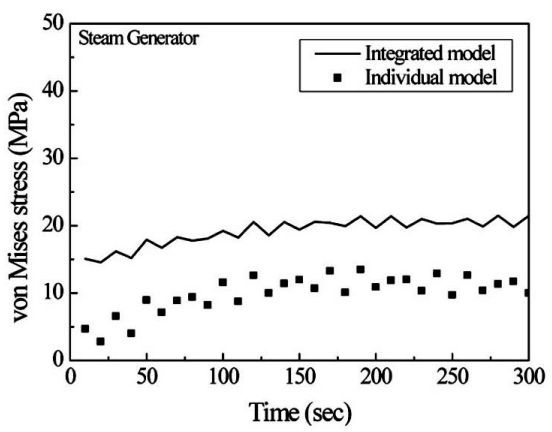

Point I

Fig. 3. Comparison Results Between Individual Models and Integrated Model 
$\mathrm{CHOl}$ et al., Fatigue Life Assessment of Reactor Coolant System Components by Using Transfer Functions of Integrated FE Model

Table 2. Difference of von Mises Effective Stresses Between Individual Models and Integrated Model

\begin{tabular}{c|c|c|c|c|c|c|c|c|c}
\hline Point & A & B & C & D & E & F & G & H & I \\
\hline Avg. Difference (\%) & 3.94 & 0.013 & 0.15 & 80.07 & 0.010 & 0.001 & 13.21 & 1.95 & 49.0 \\
\hline Max. Difference (\%) & 5.48 & 0.020 & 0.22 & 89.46 & 0.016 & 0.003 & 14.81 & 2.06 & 81.0 \\
\hline
\end{tabular}

two types of models, which did not consider external loads in the FE analyses of the individual models. Table 2 represents the differences of von Mises effective stresses between the integrated model and individual models.

As described previously, the purpose of this research is to suggest the possibility of using the integrated model for fatigue life assessment of RCS components. The model has advantages in reducing lots of repetitive transient analyses and taking into account the real disposition itself. Actually, in most locations except for geometric and/or material discontinuities, this method was successful in realizing the anticipated results. However, for the joint locations, material discontinuity and complex geometry obstructed the delineation of realistic structural behavior. To resolve these limitations, enhancing the prototype of the integrated FE model is required. Furthermore, it is worthy to consider the embodiment of the weld parts during the analysis in order to resolve material discontinuity. However, these remain as topics of future research due to the limitations of this current numerical analysis, which can be solved by employing the parallel processing technique.

\section{STRESS TRANSFER FUNCTIONS}

\subsection{Concept of Transfer Function}

The stress at an arbitrary point of a component can be separated into mechanical stress and thermal stress as follows:

$$
\sigma(t)=\sigma_{p}(t)+\sigma_{t}(t)
$$

where, $\sigma_{p}(t)$ is the mechanical stress and $\sigma_{t}(t)$ is the thermal stress.

The thermal stress can be calculated from the temperature distributions at an arbitrary time point. Eq. (2) shows that heat transfer occurs by convection and conduction at a thermal boundary and in a body.

$$
K(p) \frac{\partial}{\partial n} T(p, t)+C(p) T(p, t)=\phi(T) F(p)
$$

where, $T(p, t)$ and $\phi(t)$ are the temperatures at point $p$ at time $t$ and the bulk coolant temperature at time $t$, respectively, $n$ is the normal direction on the boundary, $K(p), C(p)$ and $F(p)$ are functions of conduction, convection and position related to the boundary conditions.

In mathematics, Green's function is used to solve inhomogeneous differential equations provided that boundary conditions are known. To calculate thermal stress, this feature was applied to engineering fields. By using Green's function, the transient temperature distribution $T(p, t)$ can be calculated by Duhamel's integral.

$$
T(p, t)=\int_{0}^{t} X(p, t-\tau) \frac{\partial}{\partial \tau} \phi(\tau) d \tau
$$

In Eq. (3), $X(p, t-\tau)$ represents Green's function of temperature. The transient thermal stress can be calculated with the given bulk temperature time history. Green's function of the thermal stress then becomes

$$
\sigma_{t}(t)=\int_{0}^{t} G(p, t-\tau) \frac{\partial}{\partial \tau} \phi(\tau) d \tau
$$

where, $G(p, t-\tau)$ is Green's function for the thermal stress at a critical location and $\phi(t)$ is the bulk coolant temperature for a transient operation. Then, Eq. (1) can be rewritten as

$$
\sigma(t)=\sigma_{p}(t)+\int_{0}^{t} G(p, t-\tau) \frac{\partial}{\partial \tau} \phi(\tau) d \tau
$$

In the above equation, Green's function is defined as the response of a system to a standard step input. Figure 4(a) represents the procedure used to develop Green's function used in this paper and Figure 4(b) illustrates the application of the proposed method by using Green's functions.

\subsection{Development of Transfer Functions}

When the unit step input of pressure was applied to a system, mechanical stress responded linearly as depicted in Figure 5(a). Therefore, a linear equation was adopted to develop the transfer function of mechanical stress. The resulting coefficient $H$ that consisted of the linear Green's function at the critical locations is summarized in Table 
Table 3. Resulting Coefficient H Related to Mechanical Stress Transfer Function

\begin{tabular}{c|c|c}
\hline Component & Critical Location & $H$ \\
\hline \multirow{4}{*}{ RPV } & Point A & 12.67 \\
\cline { 2 - 3 } & Point B & 8.38 \\
\cline { 2 - 3 } & Point C & 9.42 \\
\hline \multirow{4}{*}{ PZR } & Point D & 5.29 \\
\cline { 2 - 3 } & Point E & 6.67 \\
\cline { 2 - 3 } & Point F & 10.17 \\
\hline \multirow{3}{*}{ SG } & Point G & 7.356 \\
\cline { 2 - 3 } & Point H & 36.32 \\
\cline { 2 - 3 } & Point I & 1.45 \\
\hline
\end{tabular}

Table 4. Resulting Coefficients $a, b$ and $c$ Related to Thermal Stress Transfer Function

\begin{tabular}{c|c|c|c|c}
\hline Component & Critical Location & $a$ & $b$ & $c$ \\
\hline \multirow{4}{*}{ RPV } & Point A & -0.032 & 7.033 & 107.6 \\
\cline { 2 - 5 } & Point B & -0.0 .31 & 6.166 & 100.2 \\
\cline { 2 - 5 } & Point C & -0.043 & 8.245 & 138.2 \\
\hline \multirow{4}{*}{ PZR } & Point D & -0.014 & 1.921 & 64.68 \\
\cline { 2 - 5 } & Point E & -0.072 & 13.24 & 249.1 \\
\cline { 2 - 5 } & Point F & -0.05 & 8.906 & 165.2 \\
\hline \multirow{3}{*}{ SG } & Point G & -0.016 & 2.806 & 100.6 \\
\cline { 2 - 5 } & Point H & -0.027 & 4.035 & 15.86 \\
\cline { 2 - 5 } & Point I & -0.052 & 8.306 & 176.2 \\
\hline
\end{tabular}

Table 5. Difference of Stress Intensities Between Green's Function and Detailed FEA

\begin{tabular}{c|c|c|c|c|c|c|c|c|c}
\hline Point & A & B & C & D & E & F & G & H & I \\
\hline Avg. Difference (\%) & 2.75 & 3.01 & 3.13 & 11.98 & 1.24 & 0.59 & 25.93 & 2.94 & 7.33 \\
\hline Max. Difference (\%) & 3.41 & 4.80 & 4.84 & 16.92 & 3.30 & 3.50 & 35.17 & 10.89 & 28.90 \\
\hline
\end{tabular}

3. The transfer function of thermal stress is affected by varying the temperature history. In this paper, the Green's function of thermal stress was presented in a quadratic form, as illustrated in Figure 5(b). Table 4 summarizes the resulting coefficients $a, b$ and $c$ that consist of the quadratic Green's function at the critical locations.

\subsection{Comparison of Stress Transfer Functions}

To verify the developed Green's functions at each critical location, time dependent stress intensities derived from Green's function were compared with those derived from detailed FE analyses results. Consequently, comparison results showed good agreement in most cases. Figure 6(a) shows the contour plot of stress intensities after $t=300 \mathrm{sec}$. Comparison results between the stress intensities derived from Green's function and detailed FEA results are summarized in Table 5 and Figure 6(b).

\subsection{Discussion on The Transfer Functions}

As mentioned previously, the stress intensities derived from transfer functions matched well with those derived from detailed FE analyses in most cases. This means that Green's functions were valid, hence, the proposed methodology that employs the integrated FE model and its transfer functions is applicable. To measure the aging status, a prototype of the expert system for the integrity evaluation of NPPs, called the aging monitor, was suggested and developed in the authors' previous studies $[12,13]$. This system consists of 5 modules such as an aging alarm/ coloring monitor, an aging database, an aging document, a real-time integrity monitor and a surveillance and inspection management system. We found a possibility for extending the real-time integrity module by connecting it with the Fatigue Monitoring System (FMS). Korea Institute of Nuclear Safety (KINS) developed a monitoring system called the Computerized technical Advisory system for the Radiological Emergency (CARE) that prevents unexpected emergency situations in NPPs. This system deals with approximately 2,000 monitoring parameters that are crucial to plants operating safely. Therefore, the FMS incorporating transfer functions are being interlinked with the CARE system to determine the real-time status of age-related parameters such as stresses and cumulative usage factors under a web-environment.

\section{CONCLUSIONS}

In the present paper, an effective fatigue life assessment methodology that uses an integrated FE model and its transfer functions were suggested. First, a prototype of the integrated FE model was developed to assess realistic structural behaviors. Its validity was verified, except for 


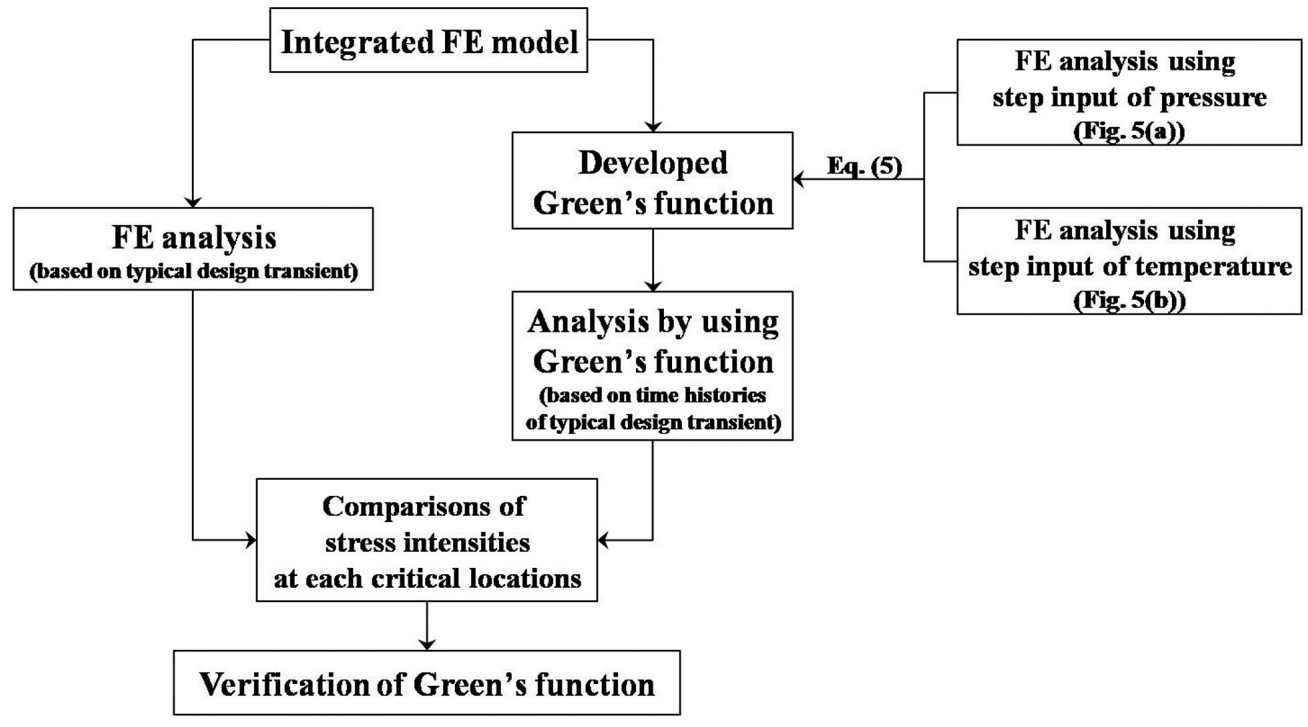

(a) Development procedure of Green's function

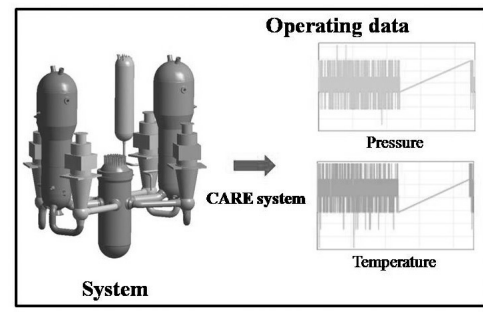

Operating data acquisition

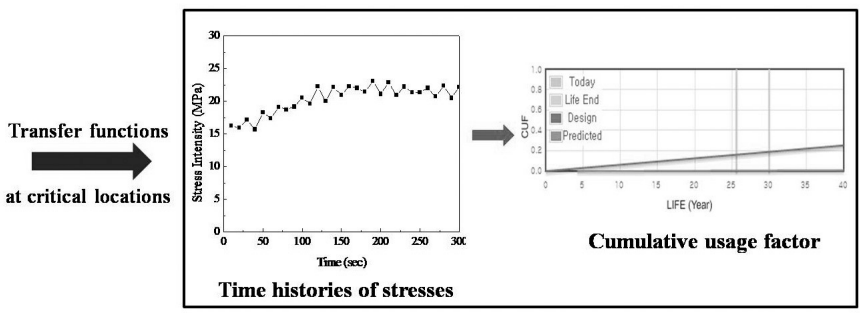

Fatigue life evaluation

(b) Application of Green's function

Fig. 4. Concept of Green's Function Development and its Application

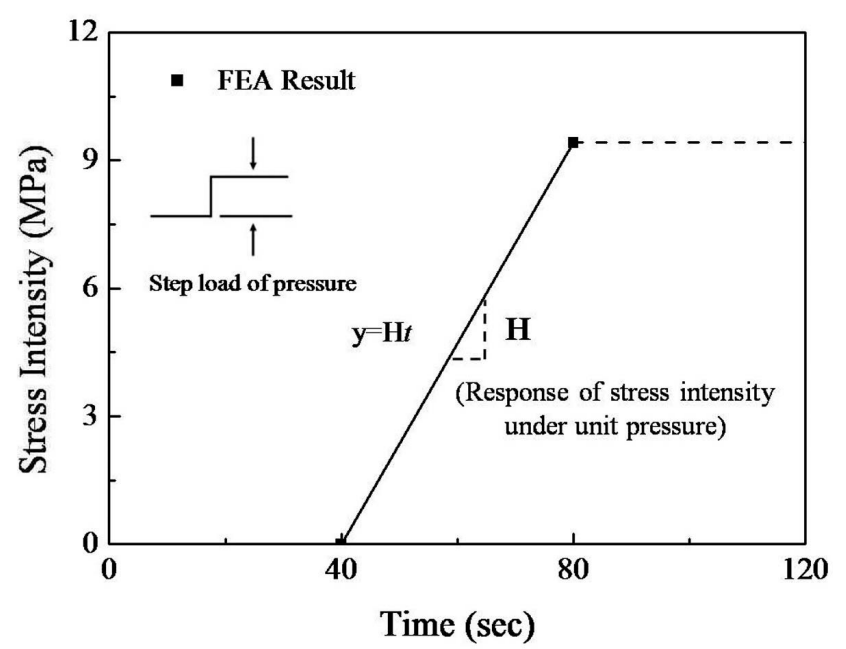

(a) Mechanical stress

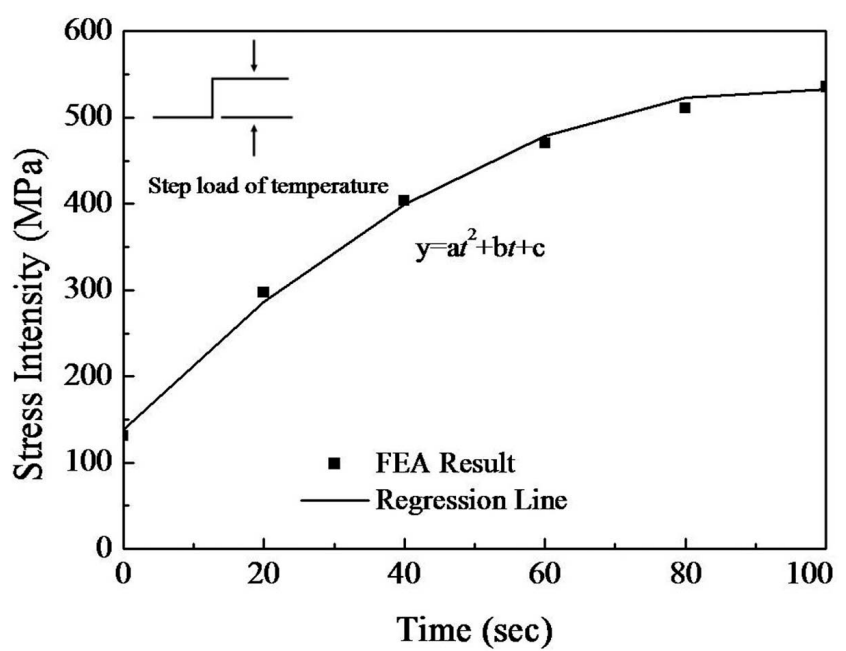

(b) Thermal stress

Fig. 5. Schematics of Transfer Functions 


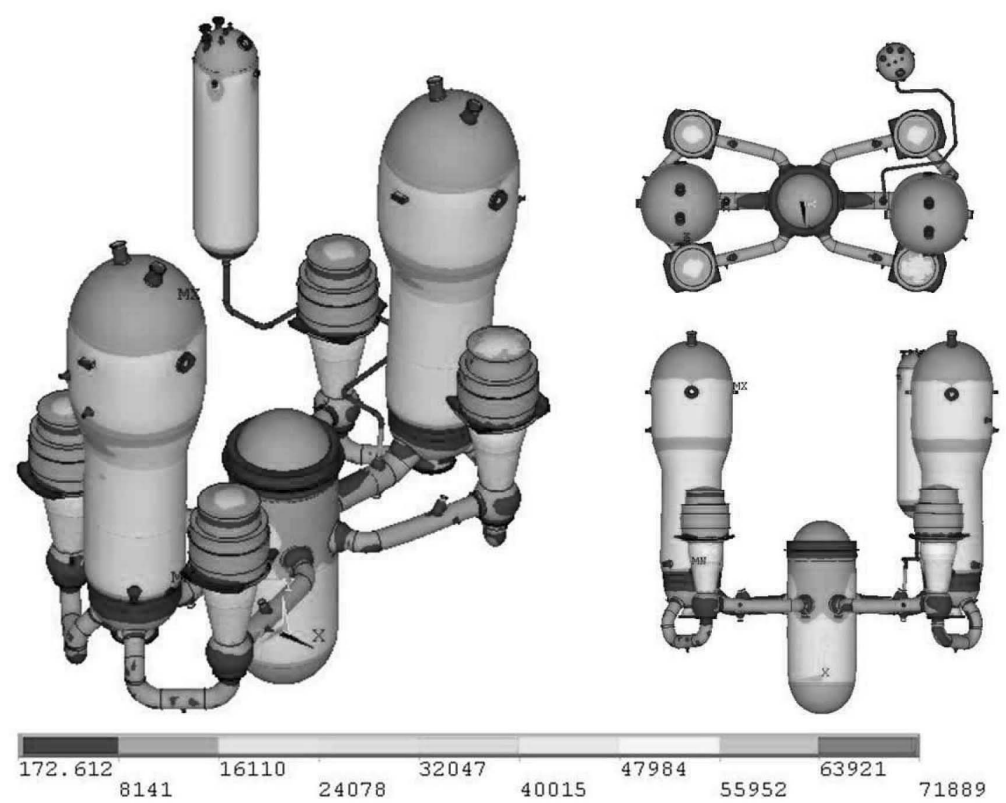

(a) Contour plot on stress intensities after $t=300 \mathrm{sec}$
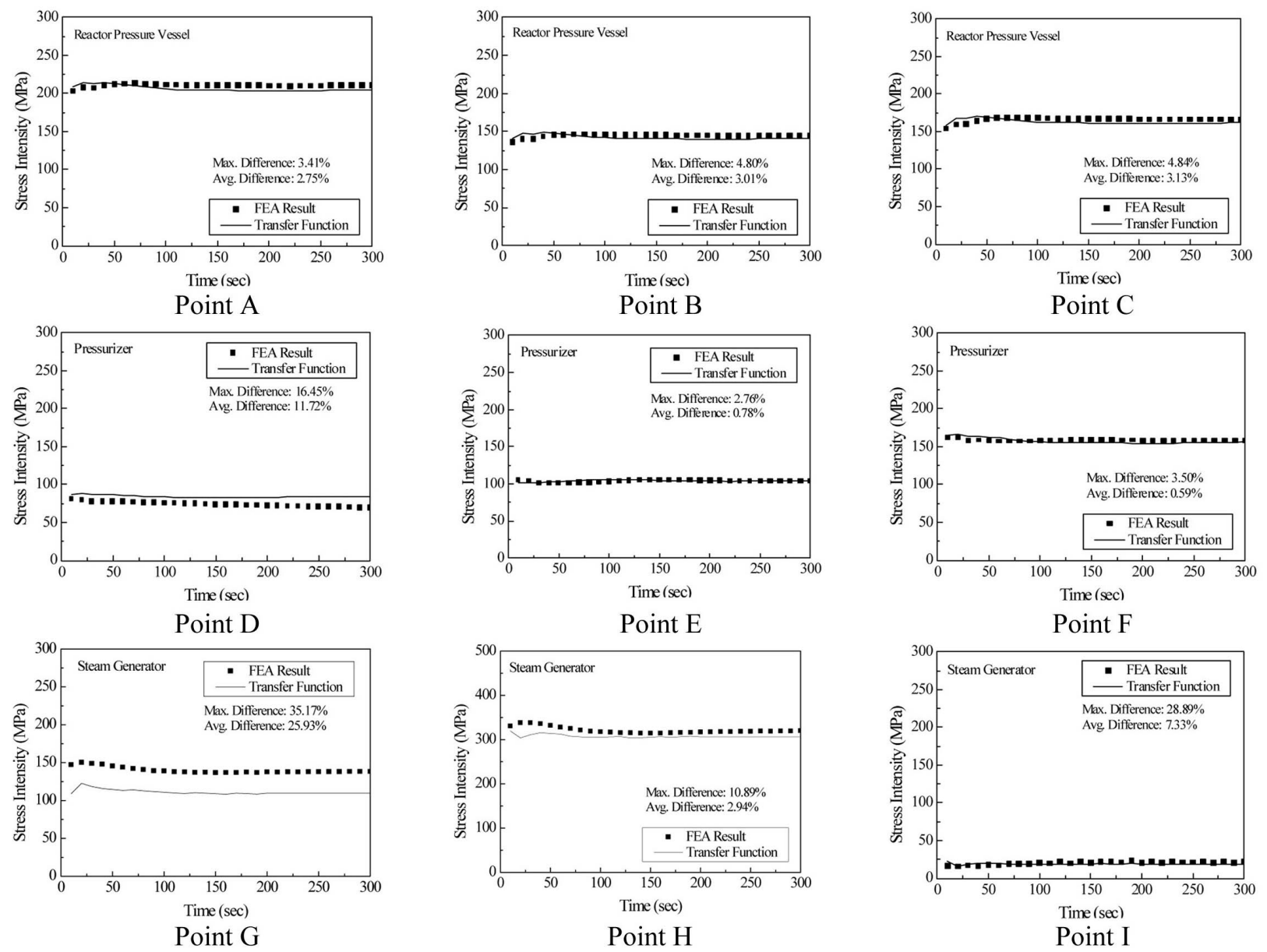

(b) Comparison of stress intensity histories

Fig. 6. Stress Intensities Derived from Green's Function and Detailed FEA Results 
the connecting points and closed points to a joint. Then mechanical and thermal stress transfer functions were derived by using the prototype of the integrated FE model with a step load at critical locations. The validation of transfer functions that were obtained was also proven. The promising transfer functions are being incorporated into the fatigue monitoring system and the possibility of connecting transfer functions with the real-time integrity monitor module is being investigated. Also, further examinations are being carried out such as weld part modeling and parallel processing for large scale analysis to maximize the applicability of the transfer functions. The proposed methodology can be useful for continued operation of existing NPPs by reducing conservatism and efforts.

\section{REFERENCES}

[ 1 ] Nuclear District Heating Reactor Development Department, "Development of Operating Transients Monitoring System for Primary Components of Nuclear Power Plants," KAERI/RR-1080/91, Korea Atomic Energy Research Institute (1991).

[2] S. Crutzen and P. Jehenson, "Inspection Performance with a View to Pressure Vessel Life Management," International Journal of Pressure Vessels and Piping, 54, 107-136 (1993).

[ 3 ] P. C. Riccardella, A. Y. Kuo, S. S. Tang and H. L. Gustin, "FatiguePro: On-Line Fatigue Usage Transient Monitoring System," EPRI NP-5835M, Electric Power Research Institute (1988).

[4] P. Aufort, G. Bimont, T. H. Chau, I. Fournier, P. Morilhat, T. Souchois and G. Codrdier, "On Line Fatiguemeter: a Large Experiment in French Nuclear Plants," Nuclear Engineering and Design, 129, 177-184 (1991).

[5] B. Yoo, "Development of Fatigue Monitoring System in Nuclear Power Plants," KAERI/RR-1307/93, Korea Atomic Energy Research Institute (1994).

[6] J. C. Kim, M. Y. Ahn, Y. S. Chang, J. B. Choi, Y. J. Kim, M. J. Jhung and Y. H. Choi, "Fatigue Life Evaluation for Nuclear Power Plant using Green's Function and Real Operating Histories," Key Engineering Materials, 326-328, 979-982 (2006).

[ 7 ] Y. S. Chang, S. B. Choi, J. B. Choi, Y. J. Kim, M. J. Jhung and Y. H. Choi, "Formulation of Three-Dimensional Green's Function and Its Application to Fatigue Life Evaluation of Pressurizer," Key Engineering Materials, 324325, 397-390 (2006).

[ 8 ] S. B. Choi, S. W. Woo, Y. S. Chang, J. B. Choi, Y. J. Kim, M. J. Jhung and Y. H. Choi, "Development of Green Functions of Steam Generator Shells using Real Operating Data," Transaction of the Korean Nuclear Society Autumn Meeting, Pyeonchang, Korea, Oct. 25-26, 2007.
[9] Sungkyunkwan University, "Study on Integrity Evaluation for Pressurized Components in Nuclear Power Plant," KINS/HR-772, Korea Institute of Nuclear Safety (2007).

[10] ANSYS, "Introduction of ANSYS Ver.11," ANSYS Inc. (2008).

[11] S. B. Choi, Y. S. Chang, J. B. Choi, Young-Jin Kim, M. J. Jhung, Y. H. Choi and T. E. Jin, "Comparative Stress Analyses of Major RCPB Components by Using a Prototype of Integrated Finite Element Model," $20^{\text {th }}$ International Conference on Structural Mechanics in Reactor Technology (SMiRT20), Espoo, Finland, August 9-14, 2009.

[12] J. B. Choi, S. W. Yeom, H. O. Ko, Y. J. Kim, H. K. Kim, Y. H. Choi and Y. W. Park, "Development of a Web-Based Aging Monitoring System for the Integrity Evaluation of Major Components in a Nuclear Power Plant," Proceedings of the 7th International Workshop on the Integrity of Nuclear Components (ASINCO7), Muju, Korea, July 3-4, 2008.

[13] Sungkyunkwan University, "Development of Web-Based Aging Evaluation Code and Web Design of Aging Monitor for Standard Nuclear Power Plant," KINS/HR-877, Korea Institute of Nuclear Safety (2008).

[14] M. Brumovsky, B. Elliot, C. Faidy, T. Inagaki, K. S. Kang, B. Kastner, T. R. Mager, Y. Makihara, J. Pachner, A. Plyushch and V. A. Piminov, "Assessment and Management of Aging of Major Nuclear Power Plant Components Important to Safety: PWR Pressure Vessels," IAEATECDOC-1120, International Atomic Energy Agency (1999).

[15] R. C. Hibbler, Mechanics of Materials, 4th ed., 409-412, Prentice Hall Inc., Upper Saddle River, New Jersey (2000).

[16] A. G. Miller, B. Kaufer and L. Carlsson, "Activities on component reliability under the OECD Nuclear Energy Agency," Nuclear Engineering Design, 198, 325-334 (2000).

[17] J. A. Bannantine, J. J. Corner and J. L. Handrock, Fundamentals of Metal Fatigue Analysis, Prentice Hall Inc., Upper Saddle River, New Jersey (1990).

[18] S. P. Timoshenko and J. N. Goodier, Theory of Elasticity, 3rd ed., McGraw-Hill International Editions, p. 68-71, 1221 Ave. of the Americas, New York (1982).

[19] F. Bazile, C. Braun, C. R. Clark, M. Condu, K. Dahlgren Persson, R. I. Facer, R. Friedrich, J. Georgiev, R. Hagen, G. Hopton, L. Langlois, M. Laraia, P. Louis, A. Omoto, N. Pieroni, A. Pryakhin, H. H. Rogner, J. Vilemas and D. J. Wilson, "Consideration of Early Closure or Continued Operation of a Nuclear Power Plant," IAEA-TECDOC1514, International Atomic Energy Agency (2006).

[20] G. H. Koo, J. J. Kwon and W. J. Kim, “Green's Function Method with Consideration of Temperature Dependant Material Properties for Fatigue Monitoring of Nuclear Power Plants," International Journal of Pressure Vessels and Piping, 86, 187-195 (2009). 